JOURNAL OF

FUNCTION SPACES AND APPLICATIONS

Volume 4, Number 1 (2006), 91-111
(C) 2006, Scientific Horizon http://www.jfsa.net

\title{
Generalized weighted Besov spaces on the Bessel hypergroup
}

\section{Miloud Assal and Hacen Ben Abdallah}

(Communicated by Jürgen Appell)

2000 Mathematics Subject Classification. 42B35.

Keywords and phrases. Bessel-Kingman hypergroup, Weight, Besov type space.

Abstract. In this paper we study generalized weighted Besov type spaces on the Bessel-Kingman hypergroup. We give different characterizations of these spaces in terms of generalized convolution with a kind of smooth functions and by means of generalized translation operators. Also a discrete norm is given to obtain more general properties on these spaces.

\section{Introduction}

The Besov spaces has been defined by many ways on $\mathbb{R}^{n}([8],[4],[14],[15]$, [12], [13]). This theory has been generalized using Hankel transform and the weight $t^{\gamma} ; \gamma>0([5],[1]$,$) . Our objective is to find weights extending$ the case $t^{\gamma}$ to define generalized weighted Besov type spaces on the BesselKingman hypergroup. In the present work we fix $\alpha>-\frac{1}{2}$ and we put $\mathbb{X}=[0,+\infty[$. For $1 \leq p, q \leq \infty$ and an adequate weight $\omega$ (see [2]) we 
define the Besov type spaces $\dot{\Lambda}_{p, q}^{\omega}(\mathbb{X})$ using the harmonic analysis associated with the Bessel operator:

$$
\left.\mathcal{L}_{\alpha}=\frac{d^{2}}{d x^{2}}+\frac{2 \alpha+1}{x} \frac{d}{d x} ; \quad x \in\right] 0, \infty[.
$$

For $\alpha=\frac{n-1}{2} ; n \in \mathbb{N}$ and $n \geq 2$, the operator $\mathcal{L}_{\alpha}$ is the radial part of the Laplacian operator defined on $\mathbb{R}^{n}$. We recall that $x \rightarrow j_{\alpha}(\lambda x)(\lambda \in[0, \infty[)$ is the unique solution of the following system (see [16])

$$
\left\{\begin{array}{l}
\mathcal{L}_{\alpha} u=-\lambda^{2} u \\
u(0)=1, \frac{d u}{d x}(0)=0
\end{array}\right.
$$

where $j_{\alpha}$ is the normalized Bessel function of first kind and order $\alpha$. The Fourier Bessel transform for a reasonable function $f$ is given by ([16]):

$$
\mathcal{F}(f)(\lambda)=\int_{\mathbb{X}} j_{\alpha}(\lambda x) f(x) d \mu_{\alpha}(x), \quad \text { for all } \lambda \in[0, \infty[
$$

where $d \mu_{\alpha}(x)=\frac{x^{2 \alpha+1} d x}{2^{\alpha} \Gamma(\alpha+1)}$.

It is well known that the Fourier-Bessel transform is a topological isomorphism from $S_{*}(\mathbb{X})$ onto itself (see [16]) where $S_{*}(\mathbb{X})$ is the Schwartz subspace consisting of even functions. This paper deals with generalized weighted Besov-Bessel type spaces defined on $\mathbb{X}$ and it is organized as follows. In the first section, we collect some harmonic analysis properties on the Bessel hypergroup which are given in [16]. In the second section we introduce the generalized weighted Besov-Bessel type spaces $\dot{\Lambda}_{p, q}^{\omega}(\mathbb{X})$, we give some properties and inclusion results of these spaces with respect to the parameters $p, q$ and $\omega$. Completeness and density results are also given in this section. We introduce an equivalent discrete norm replacing the group $] 0,+\infty\left[\right.$ by the 2 -powers group $\mathbb{D}_{2}=\left\{2^{j} ; j \in \mathbb{Z}\right\}$ to give a new characterization of the space $\dot{\Lambda}_{p, q}^{\omega}(\mathbb{X})$ and to develop other inclusion properties. In the third section we characterize the space $\Lambda_{p, q}^{\omega}(\mathbb{X})=$ $\dot{\Lambda}_{p, q}^{\omega}(\mathbb{X}) \cap L^{p}$ under some conditions on weights by equivalent norms using the difference $\Delta_{x} f=T_{x}^{(\alpha)} f-f$ and the generalized modulus of continuity $m_{p}(f, x)=\sup _{0 \leq y \leq x}\left\|\Delta_{y} f\right\|_{p}$ associated with the translation operators $T_{x}^{(\alpha)}$. In proving these results, the main tool used here is the harmonic analysis on the Bessel hypergroup.

Finally, we mention that $C$ will be always used to denote a suitable positive constant that is not necessarily the same in each occurrence. 


\section{Preliminaries}

In this section we recall some basic results in harmonic analysis related to the Bessel operators (see [16]). We first begin by some notations.

Notations. Let $\alpha>-\frac{1}{2}$ and $\mathbb{X}=[0, \infty[$. We denote by

- $\left.\mathbb{R}_{+}^{*}=\right] 0,+\infty[$.

- $\mathcal{C}_{*}(\mathbb{X})$ the space of even continuous functions on $\mathbb{R}$.

- $\mathcal{C}_{*}^{\infty}(\mathbb{X})$ the space of functions $f: \mathbb{R} \rightarrow \mathbb{C}$, even and $\mathcal{C}^{\infty}$ on $\mathbb{R}$.

- $\mathcal{D}_{*}(\mathbb{X})$ the subspace of $\mathcal{C}_{*}^{\infty}(\mathbb{X})$ consisting of functions with compact support.

- $\mathcal{C}_{*, 0}(\mathbb{X})$ the space consisting of even continuous functions tending to zero at the infinity.

- $S_{*}(\mathbb{X})$ is the Schwartz subspace consisting of even functions.

- $\mathcal{D}_{*}^{\prime}(\mathbb{X})\left(\right.$ resp. $\left.S_{*}^{\prime}(\mathbb{X})\right)$ the topological dual of $\mathcal{D}_{*}(\mathbb{X})\left(\right.$ resp. $\left.S_{*}(\mathbb{X})\right)$.

- $S_{*, 0}(\mathbb{X})$ the subspace of functions $\psi$ in $S_{*}(\mathbb{X})$ such that the support of $\mathcal{F}(\psi)$ is a compact subset of $] 0,+\infty[$.

- $S_{*, 0}^{1}(\mathbb{X})$ the subset of functions $\psi$ in $S_{*, 0}(\mathbb{X})$ such that

$$
\left.\int_{0}^{\infty}|\mathcal{F} \psi(r \lambda)|^{2} \frac{d r}{r}=1, \quad \text { for all } \lambda \in\right] 0, \infty[.
$$

These functions are known as generalized wavelets on $\mathbb{X}$.

- $L^{p}\left(d \mu_{\alpha}\right)=L^{p}\left(\mathbb{X}, d \mu_{\alpha}\right), 1 \leq p \leq \infty$, the Lebesgue space associated to the measure $d \mu_{\alpha}$ and $\|\cdot\|_{p}$ its usual norm given by:

$$
\begin{aligned}
\|f\|_{p} & =\left(\int_{\mathbb{X}}|f(x)|^{p} d \mu_{\alpha}(x)\right)^{\frac{1}{p}}, \quad \text { if } p \in[1, \infty[ \\
\|f\|_{\infty} & =\underset{x \in \mathbb{X}}{\operatorname{esssup}}|f(x)| .
\end{aligned}
$$

- $f_{r}(x)=r^{-(2 \alpha+2)} f\left(\frac{x}{r}\right) ; r>0$, the dilated of the function $f$ defined on $\mathbb{X}$ preserving the integral of $f$ with respect to the measure $d \mu_{\alpha}$, in the sense that

$$
\int_{\mathbb{X}} f_{r}(x) d \mu_{\alpha}(x)=\int_{\mathbb{X}} f(x) d \mu_{\alpha}(x), \text { for all } r>0 \text { and } f \in L^{1}\left(d \mu_{\alpha}\right) .
$$

Definition 2.1. - The generalized translation operators $T_{x}^{(\alpha)}, x \geq 0$, associated with the Bessel operators are defined for appropriate functions 
$f$ by (see [16]):

(2.2) $T_{x}^{(\alpha)} f(y)=\frac{\Gamma(\alpha+1)}{\sqrt{\pi} \Gamma\left(\alpha+\frac{1}{2}\right)} \int_{0}^{\pi} f\left(\sqrt{x^{2}+y^{2}+2 x y \cos \theta}\right)(\sin \theta)^{2 \alpha} d \theta$, for all $y \in \mathbb{X}$.

- The generalized convolution product associated with the Bessel operators is defined for a suitable pair of functions $f$ and $g$ by (see [16]):

$$
f_{\#} g(x)=\int_{\mathbb{X}} T_{x}^{(\alpha)} f(y) g(y) d \mu_{\alpha}(y), \quad \text { for all } x \in \mathbb{X} .
$$

We recall that $\left(\mathbb{X}, \#, i d_{\mathbb{X}}\right)$ is an hypergroup in the sense of Jewett ([10], [7]) called Bessel-Kingman hypergroup and we mention that, to make difference between the space variables, the dual variables and the dilation variables, we shall denote them respectively by $x \in \mathbb{X}, \lambda \in[0, \infty[$ and $r>0$.

Properties. The following results are standard in the sense that they appear in monographs on harmonic analysis like ([16]). For suitable function $f: \mathbb{X} \rightarrow \mathbb{C}$ we have

(1) $T_{0}^{(\alpha)} f(y)=f(y), \forall y \in \mathbb{X}$.

(2) $T_{x}^{(\alpha)} f(y)=T_{y}^{(\alpha)} f(x), \forall x, y \in \mathbb{X}$.

(3) $T_{x}^{(\alpha)} f(y)=\int_{\mathbb{X}} W_{\alpha}(x, y, z) f(z) d \mu_{\alpha}(z), \forall x, y \in \mathbb{X}$,

where

$$
W_{\alpha}(x, y, z)=\left\{\begin{array}{l}
\frac{2^{1-\alpha} \Gamma^{2}(\alpha+1)}{\sqrt{\pi} \Gamma\left(\alpha+\frac{1}{2}\right)} \frac{\left[(x+y)^{2}-z^{2}\right]^{\alpha-\frac{1}{2}}\left[z^{2}-(x-y)^{2}\right]^{\alpha-\frac{1}{2}}}{(x y z)^{2 \alpha}}, \\
\quad \text { if }|x-y|<z<x+y \\
0, \quad \text { otherwise. }
\end{array}\right.
$$

(4) Let $f$ be in $L^{p}\left(d \mu_{\alpha}\right), 1 \leq p \leq \infty$. Then for all $x \in \mathbb{X}$, the function $T_{x}^{(\alpha)} f$ belongs to $L^{p}\left(d \mu_{\alpha}\right)$ and we have

$$
\left\|T_{x}^{(\alpha)} f\right\|_{p} \leq\|f\|_{p}
$$

(5) For $f$ in $L^{p_{1}}\left(d \mu_{\alpha}\right)$ and $g$ in $L^{p_{2}}\left(d \mu_{\alpha}\right) ; 1 \leq p_{1}, p_{2} \leq \infty$ the function $f_{\#} g$ belongs to $L^{p_{3}}\left(d \mu_{\alpha}\right), \frac{1}{p_{1}}+\frac{1}{p_{1}}=1+\frac{1}{p_{3}}$, and we have

$$
\left\|f_{\#} g\right\|_{p_{3}} \leq\|f\|_{p_{1}}\|g\|_{p_{2}} .
$$

(6) Let $f$ be in $L^{1}\left(d \mu_{\alpha}\right)$. Then the function $\mathcal{F}(f)$ belongs to $\mathcal{C}_{*, 0}(\mathbb{X})$ and we have

$$
\|\mathcal{F}(f)\|_{\infty} \leq\|f\|_{1}
$$


where $\|\mathcal{F}(f)\|_{\infty}=\sup _{\lambda \in[0, \infty[}|\mathcal{F}(f)(\lambda)|$.

(7) Let $f$ and $g$ in $L^{1}\left(d \mu_{\alpha}\right)$, then we have

$$
\mathcal{F}\left(f_{\#} g\right)=\mathcal{F}(f) \mathcal{F}(g) .
$$

(8) Let $f$ be in $L^{1}\left(d \mu_{\alpha}\right)$. Then, for all $x \in \mathbb{X}$ and $\lambda \in[0, \infty[$, we have

$$
\mathcal{F}\left(T_{x}^{(\alpha)} f\right)(\lambda)=j_{\alpha}(\lambda x) \mathcal{F}(f)(\lambda) .
$$

Remark 2.1. Let $p \geq 1$. Then for all $f \in L^{p}\left(d \mu_{\alpha}\right)$ and $\psi \in S_{*}(\mathbb{X})$, $\langle f, \psi\rangle$ means the value of $f \in S_{*}^{\prime}(\mathbb{X})$ on $\psi$ and it is given by:

$$
\langle f, \psi\rangle=\int_{0}^{\infty} f(x) \psi(x) d \mu_{\alpha}(x)
$$

Hence, we identify $L^{p}(\mathbb{X})$ with a subspace of $S_{*}^{\prime}(\mathbb{X})$ via the formula $(2.3)$.

It might be observed that a long list of properties of the classical distributions on $\mathbb{R}^{n}$ remains valid also in our context. On the other hand it is not difficult to verify that the Fourier-Bessel transform and the Bessel operators are symmetric in the following sense

$$
\int_{0}^{\infty} f \mathcal{F}(g) d \mu_{\alpha}=\int_{0}^{\infty} \mathcal{F}(f) g d \mu_{\alpha}
$$

and

$$
\int_{0}^{\infty} \mathcal{L}_{\alpha} f g d \mu_{\alpha}=\int_{0}^{\infty} \mathcal{L}_{\alpha} g f d \mu_{\alpha}, \text { for all } f, g \in S_{*}(\mathbb{X})
$$

Therefore, we extend naturally the Fourier-Bessel transform and the operator $\mathcal{L}_{\alpha}$ on $S_{*}^{\prime}(\mathbb{X})$ as follows:

For all $T \in S_{*}^{\prime}(\mathbb{X})$ and $\psi \in S_{*}(\mathbb{X})$ we put

$$
\langle\mathcal{F}(T), \psi\rangle=\langle T, \mathcal{F}(\psi)\rangle \quad \text { and } \quad\left\langle\mathcal{L}_{\alpha} T, \psi\right\rangle=\left\langle T, \mathcal{L}_{\alpha} \psi\right\rangle
$$

It has been mentioned in [16], p. 108 that for all $f \in \mathcal{D}_{*}^{\prime}(\mathbb{X})$ (resp. $S_{*}^{\prime}(\mathbb{X})$ ) and $\psi \in \mathcal{D}_{*}(\mathbb{X})$ (resp. $S_{*}(\mathbb{X})$ ), the generalized convolution product associated with Bessel operators of $f$ and $\psi$ is the function in $\mathcal{C}_{*}^{\infty}(\mathbb{X})$ given by $\psi_{\#} f(x)=<f, T_{x}^{\alpha} \psi>$.

Definition 2.2. A weight will be a measurable function $\omega: \mathbb{R}_{+}^{*} \rightarrow \mathbb{R}_{+}^{*}$ satisfying

$$
\exists C>0 ; \quad \forall r, \rho>0 \quad\left(\frac{1}{2} \leq \frac{r}{\rho} \leq 2 \Rightarrow w(r) \leq C w(\rho)\right) .
$$


An elementary example of weight is the function defined on $\mathbb{R}_{+}^{*}$ by $w_{\gamma, \beta}(r)=r^{\gamma}(1+|\log r|)^{\beta} ; \alpha, \beta \in \mathbb{R}$.

\section{Generalized Weighted Besov-Bessel Type Spaces}

In what follows we endow the group $\mathbb{R}_{+}^{*}$ with the invariant measure $\frac{d r}{r}$. We introduce the generalized weighted Besov-Bessel type space $\dot{\Lambda}_{p, q}^{\omega}(\mathbb{X})$ and we give some properties.

Definition 3.1. Let $1 \leq p, q \leq \infty, \psi \in S_{*, 0}^{1}(\mathbb{X})$ and $\omega$ a weight. We define the generalized weighted Besov-Bessel type space $\dot{\Lambda}_{p, q}^{\omega, \psi}(\mathbb{X})$ as the set of all $f \in S_{*}^{\prime}(\mathbb{X})$ such that

$$
f=\int_{0}^{\infty} f_{\#} \psi_{r \#} \psi_{r} \frac{d r}{r}
$$

and $\mathbf{A}_{p, q}^{\omega, \psi}(f)<\infty$, where

$$
\mathbf{A}_{p, q}^{\omega, \psi}(f)= \begin{cases}\left(\int_{0}^{\infty}\left(\frac{\left\|f_{\#} \psi_{r}\right\|_{p}}{\omega(r)}\right)^{q} \frac{d r}{r}\right)^{\frac{1}{q}}, & \text { if } \quad 1 \leq q<\infty, \\ \underset{r>0}{\operatorname{esssup}}\left(\frac{\left\|f_{\#} \psi_{r}\right\|_{p}}{\omega(r)}\right), & \text { if } \quad q=\infty .\end{cases}
$$

Remark 3.1. (1) We begin by mentioning that the definition of the generalized weighted Besov-Bessel type space $\dot{\Lambda}_{p, q}^{\omega, \psi}(\mathbb{X})$ given here is the same than that introduced by Chemin in the classical case for $w(r)=r^{\gamma}$ (see [9]) which is generalized by Bahouri, Gérard and Xu on the Heisemberg group (see [6]) and by M. Assal and H. Ben Abdallah on the Laguerre hypergroup (see [3]). We do not choose the classical definition introduced by Peetre (see [12]) in which $\dot{\Lambda}_{p, q}^{\omega, \psi}(\mathbb{X})$ is defined as a set of distributions modulo polynomials.

(2) We note here that the expression (3.1) is independent, in $S_{*}^{\prime}(\mathbb{X})$, of the choice of $\psi$ in $S_{*, 0}^{1}$.

(3) If $f$ belongs to $L^{2}(\mathbb{X})$, then $(3.1)$ holds in $L^{2}(\mathbb{X})$, which is a consequence of Plancherel's formula (see [16]).

(4) The expression (3.1) is not true in $S_{*}^{\prime}(\mathbb{X})$ if $f$ is a polynomial function on $\mathbb{X}$. Indeed in this case, for all $r>0$, we have $f_{\#} \psi_{r}=0$. Thus, let $\mathbb{P}_{*}[\mathbb{X}]$ the subspace of even polynomials, then $\mathbb{P}_{*}[\mathbb{X}]$ is invariant under the generalized translation operators $T_{x}^{(\alpha)} ; x \in \mathbb{X}$ and we have $f_{\#} \psi_{r}=0$ for all $\psi \in S_{*, 0}^{1}$. To prove the above result, it suffice to take $P(y)=y^{2 k} ; k \in \mathbb{N}$. 
Then it holds

$$
\begin{aligned}
T_{x}^{(\alpha)} P(y)= & \frac{\Gamma(\alpha+1)}{\sqrt{\pi} \Gamma\left(\alpha+\frac{1}{2}\right)} \int_{0}^{\pi}\left(x^{2}+y^{2}+2 x y \cos \theta\right)^{k}(\sin \theta)^{2 \alpha} d \theta \\
= & \frac{\Gamma(\alpha+1)}{\sqrt{\pi} \Gamma\left(\alpha+\frac{1}{2}\right)} \sum_{j=0}^{k} 2^{j} \complement_{k}^{j}\left(x^{2}+y^{2}\right)^{k-j}(x y)^{j} \\
& \times \int_{0}^{\pi}(\cos \theta)^{j}(\sin \theta)^{2 \alpha} d \theta .
\end{aligned}
$$

The result follows using the fact that $\int_{0}^{\pi}(\cos \theta)^{j}(\sin \theta)^{2 \alpha} d \theta=0$ for all odd integer $j$. Now, using the fact that $\mathcal{F}(\psi)$ belongs to $S_{*, 0}(\mathbb{X})$ and the invariance of $\mathbb{P}_{*}[\mathbb{X}]$ under $T_{x}^{(\alpha)}$ we obtain $f_{\#} \psi_{r}=0$.

The following proposition gives the independence of the space $\dot{\Lambda}_{p, q}^{\omega, \psi}(\mathbb{X})$ on the choice of $\psi$ in $S_{*, 0}^{1}$.

Proposition 3.1. Let $1 \leq p, q \leq \infty$ and $\omega$ a weight. Then the space $\dot{\Lambda}_{p, q}^{\omega, \psi}(\mathbb{X})$ is independent of the choice of the function $\psi$ in $S_{*, 0}^{1}(\mathbb{X})$.

Not that the function $w(r)=r^{\gamma}$ satisfies the condition (2.4) for all $\gamma \in \mathbb{R}$.

The main idea in the proof of the above proposition is based on the following lemma.

Lemma 3.1. Let $w$ a weight. Then the following assertions are equivalent

(i) $\exists C>0 ; \forall r, \rho>0\left(\frac{1}{2} \leq \frac{r}{\rho} \leq 2 \Rightarrow w(r) \leq C w(\rho)\right)$.

(ii) For all $\alpha, \beta$ such that $0<\alpha<\beta, \exists C>0 ; \forall r, \rho>0,\left(\alpha \leq \frac{r}{\rho} \leq\right.$ $\beta \Rightarrow w(r) \leq C w(\rho))$.

Proof of Proposition 3.1. Assume $\psi$ and $\phi$ be a pair of functions belonging to $S_{*, 0}^{1}(\mathbb{X})$. To get the desired result it suffices to prove that $\dot{\Lambda}_{p, q}^{\omega, \psi}(\mathbb{X}) \subseteq$ $\dot{\Lambda}_{p, q}^{\omega, \phi}(\mathbb{X})$. Since $\mathcal{F} \phi$ and $\mathcal{F} \psi$ are supported by some compact subsets of ] $0, \infty\left[\right.$, then there exist $\alpha, \beta(0<\alpha<\beta)$ such that $\phi_{\rho \#} \psi_{r}=0$ if $\frac{r}{\rho} \notin[\alpha, \beta]$. Moreover we have $\left\|\phi_{\rho \#} \psi_{r}\right\|_{1} \leq\|\phi\|_{1}\|\psi\|_{1}$ and we obtain for all $f \in \dot{\Lambda}_{p, q}^{\omega, \psi}(\mathbb{X})$

$$
f_{\#} \phi_{\rho}=\int_{\alpha \rho}^{\beta \rho}\left(f_{\#} \psi_{r}\right)_{\#} \psi_{r \#} \phi_{\rho} \frac{d r}{r} .
$$

Using the fact that $w$ satisfies (2.4) and Lemma 3.1 we get

$$
\frac{\left\|f_{\#} \phi_{\rho}\right\|_{p}}{w(\rho)} \leq C \int_{\alpha}^{\beta} \frac{\left\|f_{\#} \psi_{r \rho}\right\|_{p}}{w(r \rho)} \frac{d r}{r}
$$


And so, Minkowski's inequality leads to

$$
\begin{aligned}
\left(\int_{0}^{\infty}\left(\frac{\left\|f_{\#} \phi_{\rho}\right\|_{p}}{w(\rho)}\right)^{q} \frac{d \rho}{\rho}\right)^{1 / q} & \leq C \int_{\alpha}^{\beta}\left(\int_{0}^{\infty}\left(\frac{\left\|f_{\#} \psi_{r \rho}\right\|_{p}}{w(r \rho)}\right)^{q} \frac{d \rho}{\rho}\right)^{1 / q} \frac{d r}{r} \\
& =C\left(\log \frac{\beta}{\alpha}\right)\left(\int_{0}^{\infty}\left(\frac{\left\|f_{\#} \psi_{\rho}\right\|_{p}}{w(\rho)}\right)^{q} \frac{d \rho}{\rho}\right)^{1 / q} .
\end{aligned}
$$

This completes the proof of the proposition.

Remark 3.2. In view of their independence of the function $\psi$ in $S_{*, 0}^{1}$ the spaces $\dot{\Lambda}_{p, q}^{\omega}(\mathbb{X}) ; 1 \leq p, q \leq \infty$ will be denoted indifferently with or without $\psi$ which will be chosen adequately.

The following proposition deals with an elementary example of weights transmitting its homogeneity to the space $\dot{\Lambda}_{p, q}^{\omega}(\mathbb{X})$.

Proposition 3.2. Let $1 \leq p, q \leq \infty$, and $\omega(\rho)=\rho^{\gamma} ;(\gamma \in \mathbb{R})$. Then the Besov-Bessel type space $\dot{\Lambda}_{p, q}^{\omega}(\mathbb{X})$ is homogeneous of degree $d(p, \gamma)=$ $\frac{2 \alpha+2}{p}-\gamma$ in the sense that, for all $f \in \dot{\Lambda}_{p, q}^{\omega}(\mathbb{X})$

$$
\mathbf{A}_{p, q}^{\omega}\left(d_{r} f\right)=r^{\frac{2 \alpha+2}{p}-\gamma} \mathbf{A}_{p, q}^{\omega}(f), \quad \text { for all } r>0
$$

where $d_{r} f$ is the distribution given by $\left\langle d_{r} f, \psi\right\rangle=\left\langle f, \psi_{1 / r}\right\rangle$ for all $\psi \in$ $S_{*}(\mathbb{X})$.

Proof. Let $1 \leq p, q \leq \infty$ and $\gamma \in \mathbb{R}$. Assume $f$ in $\dot{\Lambda}_{p, q}^{\omega}(\mathbb{X})$, then

$$
\begin{aligned}
\mathbf{A}_{p, q}^{\omega}\left(d_{r} f\right) & =\left\|\frac{\left\|\left(d_{r} f\right)_{\#} \psi_{\rho}\right\|_{p}}{\rho^{\gamma}}\right\|_{L^{q}\left(\frac{d \rho}{\rho}\right)} \\
& =r^{\frac{2 \alpha+2}{p}}\left\|\frac{\left\|f_{\#} \psi_{\frac{\rho}{r}}\right\|_{p}}{\rho^{\gamma}}\right\|_{L^{q}\left(\frac{d \rho}{\rho}\right)} \\
& =r^{\frac{2 \alpha+2}{p}-\gamma}\left\|\frac{\left\|f_{\#} \psi_{\rho}\right\|_{p}}{\rho^{\gamma}}\right\|_{L^{q}\left(\frac{d \rho}{\rho}\right)} \\
& =r^{\frac{2 \alpha+2}{p}-\gamma} \mathbf{A}_{p, q}^{\omega}(f) .
\end{aligned}
$$

Definition 3.2. Let $1 \leq p, q, q^{\prime} \leq \infty ; \frac{1}{q^{\prime}}+\frac{1}{q}=1$. We denote by $W_{\frac{2 \alpha+2}{p}}$ the class of weights satisfying

(i) $w(r) r^{\frac{-(2 \alpha+2)}{p}}$ belongs to $L^{q^{\prime}}\left(\left[1,+\infty\left[, \frac{d r}{r}\right)\right.\right.$.

(ii) There exists $\varepsilon>0$ such that $w(r) r^{\varepsilon}$ belongs to $L^{q^{\prime}}\left([0,1], \frac{d r}{r}\right)$.

The following proposition holds. 
Proposition 3.3. Let $1 \leq p, q \leq \infty$ and $\omega \in W_{\frac{2 \alpha+2}{p}}$. Then $\dot{\Lambda}_{p, q}^{\omega}(\mathbb{X})$ is a Banach space.

Remark that the weight $w(r)=r^{\gamma}$ belongs to $W_{\frac{2 \alpha+2}{p}}$ for $\gamma<\frac{2 \alpha+2}{p}$ which coincides with the classical case. To prove the above proposition we need the following lemmas.

Lemma 3.2. Let $h \in S_{*}(\mathbb{X})$ and $\psi \in S_{*, 0}^{1}(\mathbb{X})$. Then, for all $k \in \mathbb{N}$, there exists $\psi_{[k]} \in S_{*, 0}(\mathbb{X})$ such that

$$
h_{\#} \psi_{r}=r^{2 k}\left(\mathcal{L}_{\alpha}^{k} h\right)_{\#}\left(\psi_{[k]}\right)_{r}
$$

where $\mathcal{L}_{\alpha}$ is the differential operator given in the introduction part. Furthermore there exists $C>0$ such that

$$
\left\|h_{\#} \psi_{r}\right\|_{1} \leq C r^{2 k} \quad \text { for all } 0 \leq r \leq 1
$$

and

$$
\left\|h_{\#} \psi_{r}\right\|_{1} \leq C \quad \text { for all } r \geq 1 .
$$

Proof. We obtain (3.2) by induction on $k$. The inequalities (3.3) and (3.4) follow from (3.2).

Lemma 3.3. Let $1 \leq p, q \leq \infty$ and $\omega \in W_{\frac{2 \alpha+2}{p}}$. For $\phi$ in $S_{*, 0}^{1}$, put

$$
\Phi(g)=\int_{0}^{\infty} w(r) g(r) \# \phi_{r} \frac{d r}{r} .
$$

Then $\Phi$ define a linear and continuous mapping from $L^{q}\left(\mathbb{R}_{+}^{*}, L^{p}(\mathbb{X}), \frac{d r}{r}\right)$ to $\dot{\Lambda}_{p, q}^{\gamma}(\mathbb{X})$.

Proof. Let us first prove that, for $g \in L^{q}\left(\mathbb{R}_{+}^{*}, L^{p}(\mathbb{X}), \frac{d r}{r}\right), \Phi(g)$ define an element of $S_{*}^{\prime}(\mathbb{X})$, that is for all $h \in S_{*}(\mathbb{X})$,

$$
\int_{0}^{\infty}\left|w(r)\left\langle g(r)_{\#} \phi_{r}, h\right\rangle\right| \frac{d r}{r}<\infty .
$$

Take $\psi \in S_{*}(\mathbb{X})$ such that $\mathcal{F}(\psi)=1$ on $\operatorname{Supp} \mathcal{F} \phi$. Then, using Hölder's and Young's inequalities, we obtain

$$
\begin{aligned}
\mid\left\langle g(r)_{\#} \phi_{r}, h>\right| & =\left|<g(r)_{\#} \phi_{r}, h_{\#} \psi_{r}\right\rangle \mid \\
& \leq\left\|g(r)_{\#} \phi_{r}\right\|_{\infty}\left\|h_{\#} \psi_{r}\right\|_{1} \\
& \leq C r^{-\frac{2 \alpha+2}{p}}\|g(r)\|_{p}\left\|h_{\#} \psi_{r}\right\|_{1} .
\end{aligned}
$$


On the other hand, using Lemma 3.2, we get

$$
\begin{aligned}
\int_{0}^{\infty}\left|w(r)<g(r)_{\#} \phi_{r}, h>\right| \frac{d r}{r} \leq & C\left\{\int_{0}^{1} w(r) r^{2 k-\frac{2 \alpha+2}{p}}\|g(r)\|_{p} \frac{d r}{r}\right. \\
& \left.+\int_{1}^{\infty} w(r) r^{-\frac{2 \alpha+2}{p}}\|g(r)\|_{p}\right\} \frac{d r}{r} \\
\leq & C\left(\int_{0}^{1}\|g(r)\|_{p}^{q} \frac{d r}{r}\right)^{1 / q} \\
\times & \left(\int_{0}^{1}\left(w(r) r^{2 k-\frac{2 \alpha+2}{p}}\right)^{\bar{q}} \frac{d r}{r}\right)^{1 / \bar{q}} \\
& +C\left(\int_{1}^{\infty}\|g(r)\|_{p}^{q} \frac{d r}{r}\right)^{1 / q} \\
& \times\left(\int_{1}^{\infty}\left(w(r) r^{-\frac{2 \alpha+2}{p}}\right)^{\frac{\bar{q}}{r}} \frac{d r}{r}\right)^{1 / \bar{q}} .
\end{aligned}
$$

where $\bar{q}$ is the conjugate exponent of $q$. Using the fact that $w$ belongs to $W_{\frac{2 \alpha+2}{p}}$, we obtain for $k$ sufficiently large

$$
\int_{0}^{\infty}\left|w(r)<g(r)_{\#} \phi_{r}, h>\right| \frac{d r}{r} \leq C\|g\|_{L^{q}\left(\mathbb{R}_{+}^{*}, L^{p}(\mathbb{X}), \frac{d r}{r}\right)}<\infty .
$$

Now, let $\psi$ in $S_{*, 0}^{1}$. From Minkowski's inequality and Young's inequality we have

$$
\begin{aligned}
\frac{\left\|\Phi(g)_{\#} \psi_{\rho}\right\|_{p}}{w(\rho)} & \leq C \int_{\alpha}^{\beta} w(r \rho) \frac{\|g(r \rho)\|_{p}}{w(\rho)} \frac{d r}{r} \\
& =C \int_{0}^{\infty} \mathbb{1}_{[\alpha, \beta]}(r)\|g(r \rho)\|_{p} \frac{d r}{r} \\
& =C(H \star G)(\rho)
\end{aligned}
$$

with $H(s)=\mathbb{1}_{[\alpha, \beta]}(s), G(s)=\|g(s)\|_{p}$ and $H \star G$ is the convolution of $H$ and $G$ on the group $\left(\mathbb{R}_{+}^{*}, \frac{d r}{r}\right)$. Now, by Young's inequality, it holds

$$
\begin{aligned}
\|\Phi(g)\|_{\hat{\Lambda}_{p, q}^{w}(\mathbb{X})} & \leq C\|H \star G\|_{L^{q}\left(\mathbb{R}_{+}, \frac{d \rho}{\rho}\right)} \\
& \leq C\|H\|_{L^{1}\left(\mathbb{R}_{+}, \frac{d \rho}{\rho}\right)}\|G\|_{L^{q}\left(\mathbb{R}_{+}, \frac{d \rho}{\rho}\right)} \\
& =C\|g\|_{L^{q}\left(\mathbb{R}_{+}^{*}, L^{p}(\mathbb{X}), \frac{d r}{r}\right)} .
\end{aligned}
$$

The lemma is proved. 
Proof of Proposition 3.3. Let $\psi$ in $S_{*, 0}^{1}$ and take $\phi=\psi$ in Lemma 3.3. Then $\Phi$ defined by (3.5) is a continuous linear mapping from $L^{q}\left(\mathbb{R}_{+}^{*}, L^{p}(\mathbb{X}), \frac{d r}{r}\right)$ to $\dot{\Lambda}_{p, q}^{\gamma}(\mathbb{X})$. On the other hand the operator $\Psi$ associating to $f$ in $\dot{\Lambda}_{p, q}^{\gamma}(\mathbb{X})$ the function $\Psi(f)$ defined on $\mathbb{R}_{+}^{*}$ by:

$$
\Psi(f)(r)=\frac{f_{\#} \psi_{r}}{w(r)}
$$

is obviously a linear isometry from $\dot{\Lambda}_{p, q}^{\gamma}(\mathbb{X})$ to $L^{q}\left(\mathbb{R}_{+}^{*}, L^{p}(\mathbb{X}), \frac{d r}{r}\right)$ and using the decomposition (3.1), we obtain $\Phi \circ \Psi=I d_{\boldsymbol{\Lambda}_{p, q}^{\gamma}(\mathbb{X})}$. This implies

$$
(\Psi \circ \Phi) \circ \Psi=\Psi \quad \text { on } \dot{\Lambda}_{p, q}^{\gamma}(\mathbb{X}) .
$$

So $\Psi\left(\dot{\Lambda}_{p, q}^{\gamma}(\mathbb{X})\right)=\operatorname{Ker}\left(\Psi \circ \Phi-I d_{L^{q}\left(\mathbb{R}_{+}^{*}, L^{p}(\mathbb{X}), \frac{d r}{r}\right)}\right)$ is a closed subspace of $L^{q}\left(\mathbb{R}_{+}^{*}, L^{p}(\mathbb{X}), \frac{d r}{r}\right)$. Since $\Psi$ is an isometry, then $\dot{\Lambda}_{p, q}^{\gamma}(\mathbb{X})$ can be identified with a closed subspace of $L^{q}\left(\mathbb{R}_{+}^{*}, L^{p}(\mathbb{X}), \frac{d r}{r}\right)$. The completeness of $\dot{\Lambda}_{p, q}^{\gamma}(\mathbb{X})$ follows.

Proposition 3.4. Let $1 \leq p \leq \infty, 1 \leq q<\infty$ and $\omega$ a weight. Then the subspace $\dot{\Lambda}_{p, q}^{\omega}(\mathbb{X}) \cap \mathcal{C}_{*}^{\infty}(\mathbb{X})$ is dense in $\dot{\Lambda}_{p, q}^{\omega}(\mathbb{X})$.

Proof. Let $\phi \in S_{*, 0}^{1}(\mathbb{X})$ and $f \in \dot{\Lambda}_{p, q}^{\gamma}(\mathbb{X})$. Then for $\varepsilon>1$, the function

$$
f_{\varepsilon}=\int_{1 / \varepsilon}^{\varepsilon} f_{\#} \phi_{r \#} \phi_{r} \frac{d r}{r}
$$

is obviously $\mathcal{C}^{\infty}$ and belongs to $\dot{\Lambda}_{p, q}^{\gamma}(\mathbb{X})$. Moreover the same reasoning given in Proposition 3.1 leads to

$$
\left\|f_{\varepsilon}-f\right\|_{\Lambda_{p, q}^{\omega}(\mathbb{X})} \leq C\left\|\frac{\left\|f_{\#} \phi_{r}\right\|_{p}}{r^{\gamma}} \mathbb{1}_{\varepsilon}(r)\right\|_{L^{q\left(\mathbb{R}_{+}, \frac{d r}{r}\right)}}
$$

where $\mathbb{1}_{\varepsilon}$ is the characteristic function of the set $\mathbb{R} \backslash[1 / \varepsilon, \varepsilon]$. And the right hand side of the above inequality tends to zero as $\varepsilon$ tends to $\infty$.

Proposition 3.5. Let $1 \leq q \leq \infty$. Then, for $1 \leq p_{1} \leq p_{2} \leq \infty$ and $\omega_{1}, \omega_{2}$ two weights such that $r^{\frac{2 \alpha+2}{p_{1}}}\left(\omega_{1}(r)\right)^{-1}=r^{\frac{2 \alpha+2}{p_{2}}}\left(\omega_{2}(r)\right)^{-1}$, we have

$$
\dot{\Lambda}_{p_{1}, q}^{\omega_{1}}(\mathbb{X}) \subseteq \dot{\Lambda}_{p_{2}, q}^{\omega_{2}}(\mathbb{X}) \quad \text { (with continuous embedding). }
$$

Proof. Let $\psi$ in $S_{*, 0}^{1}$ and let $1 \leq p_{3} \leq \infty$ such that $\frac{1}{p_{1}}+\frac{1}{p_{3}}=1+\frac{1}{p_{2}}$. We consider $\phi \in S_{*}(\mathbb{X})$ satisfying $\mathcal{F} \phi=1$ on $\operatorname{supp}(\mathcal{F} \psi)$. Then we obtain 
for all $r>0$ and $f \in \dot{\Lambda}_{p_{1}, q}^{\omega_{1}}(\mathbb{X})$

$$
\begin{aligned}
\left\|f_{\#} \psi_{r}\right\|_{p_{2}} & =\left\|f_{\#} \psi_{r \#} \phi_{r}\right\|_{p_{2}} \\
& \leq\left\|f_{\#} \psi_{r}\right\|_{p_{1}}\left\|\phi_{r}\right\|_{p_{3}} \\
& =C\left\|f_{\#} \psi_{r}\right\|_{p_{1}} r^{(2 \alpha+2)\left(\frac{1}{p_{3}}-1\right)}
\end{aligned}
$$

So, it holds

$$
\frac{\left\|f_{\#} \psi_{r}\right\|_{p_{2}}}{\omega_{2}(r)} \leq C \frac{\left\|f_{\#} \psi_{r}\right\|_{p_{1}}}{\omega_{1}(r)} .
$$

Which leads to $\mathbf{A}_{p_{2}, q}^{\omega_{2}}(f) \leq C \mathbf{A}_{p_{1}, q}^{\omega_{1}}(f)$.

To obtain more general properties we introduce an equivalent discrete norm on $\dot{\Lambda}_{p, q}^{\omega}(\mathbb{X})$ replacing the group $\mathbb{R}_{+}^{*}$ by the 2 -powers group $\mathbb{D}_{2}=$ $\left\{2^{j} ; j \in \mathbb{Z}\right\}$ equipped with the invariant counting measure.

Theorem 3.1. Let $1 \leq p, q \leq \infty, \omega$ aweight and $\theta \in S_{*, 0}(\mathbb{X})$ such that $\mathcal{F} \theta \in \mathcal{D}_{*}(] 0, \infty[)$ and, for fixed $\lambda_{2}>2 \lambda_{1}>0, \mathcal{F} \theta(\lambda) \neq 0$ on $\left[\lambda_{1}, \lambda_{2}\right]$. For $f$ in $\dot{\Lambda}_{p, q}^{\omega}(\mathbb{X})$ put

$$
\mathbf{D}_{p, q}^{\omega, \theta}(f)= \begin{cases}\left(\sum_{j \in \mathbb{Z}}\left(\frac{\left\|f_{\#} \theta_{2^{j}}\right\|_{p}}{\omega\left(2^{j}\right)}\right)^{q}\right)^{\frac{1}{q}}, & \text { if } \quad 1 \leq q<\infty, \\ \sup _{j \in \mathbb{Z}}\left(\frac{\left\|f_{\#} \theta_{2^{j}}\right\|_{p}}{\omega\left(2^{j}\right)}\right), & \text { if } \quad q=\infty .\end{cases}
$$

Then $\mathbf{D}_{p, q}^{\omega, \theta}$ is a norm on $\dot{\Lambda}_{p, q}^{\omega}(\mathbb{X})$ equivalent to the norm $\mathbf{A}_{p, q}^{\omega}$.

Remark 3.3. For $\omega$ a weight, two different functions $\theta_{1}$ and $\theta_{2}$ satisfying the hypothesis of the above theorem give two equivalent norms.

Proof of the Theorem 3.1. To obtain the desired result we will compare the norms $\mathbf{A}_{p, q}^{\omega}$ and $\mathbf{D}_{p, q}^{\omega}$. Using the fact that $\mathcal{F} \theta \neq 0$ on $\left[\lambda_{1}, \lambda_{2}\right]$ for $\lambda_{2}>2 \lambda_{1}>0$, there exists $\mathcal{F} \sigma$ belonging to $\mathcal{D}_{*}(] 0, \infty[)$ such that $(\mathcal{F} \theta)(\mathcal{F} \sigma)=1$ on $\left[\lambda_{1}, \lambda_{2}\right]$. Let $\psi \in S_{*, 0}^{1}$ satisfying $\operatorname{supp}(\mathcal{F} \psi) \subset\left[2 \lambda_{1}, \lambda_{2}\right]$. This gives, for all $1 \leq r \leq 2$ and $\lambda \in[0, \infty[$

$$
\mathcal{F} \psi\left(2^{j} r \lambda\right)=\mathcal{F} \psi\left(2^{j} r \lambda\right) \mathcal{F} \theta\left(2^{j} \lambda\right) \mathcal{F} \sigma\left(2^{j} \lambda\right) .
$$

Since $\omega$ satisfies (2.4), it holds for $1 \leq q<\infty$ that

$$
\begin{aligned}
\mathbf{A}_{p, q}^{\omega}(f) & =\left(\sum_{j \in \mathbb{Z}} \int_{1}^{2}\left(\frac{\left\|f_{\#} \psi_{2^{j} r}\right\|_{p}}{\omega\left(r 2^{j}\right)}\right)^{q} \frac{d r}{r}\right)^{\frac{1}{q}} \\
& \leq C\left(\sum_{j \in \mathbb{Z}} \int_{1}^{2}\left(\frac{\left\|f_{\#} \theta_{2^{j}}\right\|_{p}}{\omega\left(2^{j}\right)}\right)^{q} \frac{d r}{r}\right)^{\frac{1}{q}}=C \log (2) \mathbf{D}_{p, q}^{\omega}(f) .
\end{aligned}
$$


Conversely, let $\mathcal{F} \theta$ supported by $\left[\lambda_{1}, \lambda_{2}\right] ; 0<\lambda_{1}<\lambda_{2}$ and let $\psi \in S_{*, 0}^{1}(\mathbb{X})$ satisfying $\mathcal{F} \psi=1$ on $\left[\lambda_{1} / 2, \lambda_{2}\right]$. Then it holds, for $1 / 2 \leq r \leq 1$

$$
\mathcal{F} \theta\left(2^{j} \lambda\right)=\mathcal{F} \psi\left(2^{j} r \lambda\right) \mathcal{F} \theta\left(2^{j} \lambda\right) \quad \text { for all } \lambda \in[0, \infty[.
$$

The same reasoning as above leads to

$$
\mathbf{D}_{p, q}^{\omega}(f) \leq C \mathbf{A}_{p, q}^{\omega}(f) .
$$

For the case $q=\infty$, assume $f \in \dot{\Lambda}_{p, \infty}^{\omega}(\mathbb{X})$ such that $\mathbf{D}_{p, \infty}^{\omega}(f)<\infty$ and let $r>0$ and $j \in \mathbb{Z}$ such that $2^{j} \leq r \leq 2^{j+1}$, then, from (3.6), we get

$$
\left\|f_{\#} \psi_{r}\right\|_{p} \leq C\left\|f_{\#} \theta_{2^{j}}\right\|_{p} \leq C \mathbf{D}_{p, \infty}^{\omega}(f) \omega\left(2^{j}\right) \leq C \mathbf{D}_{p, \infty}^{\omega}(f) \omega(r)
$$

which implies that $\mathbf{A}_{p, \infty}^{\omega}(f) \leq C \mathbf{D}_{p, \infty}^{\omega}(f)$.

Conversely let us take $f \in \dot{\Lambda}_{p, \infty}^{\omega}(\mathbb{X})$. Then, from (3.7) we obtain for $1 / 2 \leq r \leq 1$ the following estimation

$$
\left\|f_{\#} \theta_{2^{j}}\right\|_{p} \leq C\left\|f_{\#} \psi_{2^{j} r}\right\|_{p} \leq C \mathbf{A}_{p, \infty}^{\omega}(f) \omega\left(2^{j} r\right) \leq C \mathbf{A}_{p, \infty}^{\omega}(f) \omega\left(2^{j}\right) .
$$

This completes the proof of the theorem.

Proposition 3.6. Let $1 \leq p \leq \infty$ and $\omega$ a weight. Then, for $1 \leq q_{1} \leq q_{2}<\infty$ we have

$$
\dot{\Lambda}_{p, q_{1}}^{\omega}(\mathbb{X}) \subseteq \dot{\Lambda}_{p, q_{2}}^{\omega}(\mathbb{X}) \quad \text { (with continuous embedding). }
$$

Proof. The result holds using the discrete norm and the fact that $l^{q_{1}} \subset l^{q_{2}}$ for all $1 \leq q_{1} \leq q_{2} \leq \infty$ and we have

$$
\left(\sum\left|u_{j}\right|^{q_{2}}\right)^{1 / q_{2}} \leq\left(\sum\left|u_{j}\right|^{q_{1}}\right)^{1 / q_{1}}
$$

for all $\left(u_{j}\right) \in l^{q_{1}}$.

\section{Characterization of the Spaces $\Lambda_{p, q}^{\omega}(\mathbb{X})=\dot{\Lambda}_{p, q}^{\omega}(\mathbb{X}) \cap L^{p}$}

In this section we study the spaces $\Lambda_{p, q}^{\omega}(\mathbb{X})=\dot{\Lambda}_{p, q}^{\omega}(\mathbb{X}) \cap L^{p}\left(d \mu_{\alpha}\right)$ endowed with the norm $\mathbf{A}_{p, q}^{\omega}$ and we give some characterizations using equivalent norms for some classes of weights $\omega$. Let us first start with the following definition.

Definition 4.1. Let $0 \leq \varepsilon, \delta<\infty, 1 \leq q, q^{\prime} \leq \infty ; \frac{1}{q^{\prime}}+\frac{1}{q}=1$ and $\omega$ be a weight. 
(i) $\omega$ is said to be a $d_{\varepsilon}$-weight if there exists $C>0$ such that

$$
\int_{0}^{s} r^{\varepsilon} \omega(r) \frac{d r}{r} \leq C s^{\varepsilon} \omega(s), \quad \text { for all } s>0 .
$$

(ii) $\omega$ is said to be a $b_{\delta}$-weight if there exists $C>0$ such that

$$
\int_{s}^{\infty} \frac{\omega(r)}{r^{\delta}} \frac{d r}{r} \leq C \frac{\omega(s)}{s^{\delta}}, \quad \text { for all } s>0 .
$$

If $\left(d_{\varepsilon}\right)$ (resp. $\left.\left(b_{\delta}\right)\right)$ denotes the class of $d_{\varepsilon}$-weight (resp. $b_{\delta}$-weight) we write

$$
\mathcal{W}_{\varepsilon, \delta}=\left(d_{\varepsilon}\right) \cap\left(b_{\delta}\right)
$$

and

$$
\mathcal{W}_{\varepsilon, \delta}^{q}=\left\{\omega: \text { weight } / \omega(r)=\chi^{\frac{1}{q^{r}}}(r) \nu^{-\frac{1}{q}}\left(r^{-1}\right) ; \chi, \nu \in \mathcal{W}_{\varepsilon, \delta}\right\} .
$$

The following properties hold

P1. If $\omega \in \mathcal{W}_{\varepsilon, \delta}^{q}$ then $\omega \in \mathcal{W}_{\varepsilon^{\prime}, \delta^{\prime}}^{q}$, for any $\varepsilon^{\prime}>\varepsilon$ and $\delta^{\prime}>\delta$.

P2. Let $\bar{\omega}(r)=\omega\left(r^{-1}\right)$. Then $\omega \in\left(b_{\varepsilon}\right) \quad$ if and only if $\quad \bar{\omega} \in\left(d_{\varepsilon}\right)$.

Note that, $w(r)=r^{\gamma}$ belongs to $\mathcal{W}_{0,2}^{q}$ for $0<\gamma<2$.

Theorem 4.1 (First characterization theorem). Let $1 \leq p, q \leq \infty$ and $\omega$ a weight belonging to $W_{0,2}^{q}$. For $f$ in $\Lambda_{p, q}^{\omega}(\mathbb{X})$ put

$$
\mathbf{B}_{p, q}^{\omega}(f)=\left\{\begin{array}{lll}
\left(\int_{\mathbb{X}}\left(\frac{\left\|\Delta_{x} f\right\|_{p}}{\omega(x)}\right)^{q} \frac{d x}{x}\right)^{\frac{1}{q}}, & \text { if } & 1 \leq q<\infty, \\
\underset{x \in \mathbb{X}}{\operatorname{esssup}\left(\frac{\left\|\Delta_{x} f\right\|_{p}}{\omega(x)}\right),} & \text { if } & q=\infty
\end{array}\right.
$$

where $\Delta_{x} f=T_{x}^{(\alpha)} f-f$, for all $x \in \mathbb{X}$. Then $\mathbf{B}_{p, q}^{\omega}$ is a norm on $\Lambda_{p, q}^{\omega}(\mathbb{X})$ equivalent to $\mathbf{A}_{p, q}^{\omega}$.

Remark 4.1. Note that the case $1<q<\infty$ could have been shown by interpolation with the extreme cases $(q=1$ and $q=\infty)$, but a direct proof is presented in this paper.

Let us first start with some basic lemmas that will be useful for our purposes.

Lemma A (Schur lemma) (see [2]). Let $1<q<\infty$ and $q^{\prime}$ its conjugate exponent. Let $\left(\Omega_{1}, \mathcal{M}_{1}, \mu_{1}\right)$ and $\left(\Omega_{2}, \mathcal{M}_{2}, \mu_{2}\right)$ be a pair of $\sigma$-finite measure spaces and let $F: \Omega_{1} \times \Omega_{2} \rightarrow \mathbb{R}_{+}$be a measurable function. Define $T_{F} f$ 
for all measurable positive function $f$ on $\Omega_{1}$ by

$$
T_{F} f\left(\omega_{2}\right)=\int_{\Omega_{1}} F\left(\omega_{1}, \omega_{2}\right) f\left(\omega_{1}\right) d \mu_{1}\left(\omega_{1}\right), \quad \text { for all } \omega_{2} \in \Omega_{2} \text {. }
$$

If there exist $C>0$ and measurable functions $\left.h_{i}: \Omega_{i} \rightarrow\right] 0,+\infty[;(i=1,2)$ such that

$$
\begin{gathered}
\int_{\Omega_{1}} F\left(\omega_{1}, \omega_{2}\right) h_{1}^{q^{\prime}}\left(\omega_{1}\right) d \mu_{1}\left(\omega_{1}\right) \leq C h_{2}^{q^{\prime}}\left(\omega_{2}\right) \quad \mu_{2}-\text { a.e. } \\
\int_{\Omega_{2}} F\left(\omega_{1}, \omega_{2}\right) h_{2}^{q}\left(\omega_{2}\right) d \mu_{2}\left(\omega_{2}\right) \leq C h_{1}^{q}\left(\omega_{1}\right) \quad \mu_{1}-\text { a.e. }
\end{gathered}
$$

Then $T_{F}$ can be extended as a bounded operator from $L^{q}\left(\Omega_{1}, \mu_{1}\right)$ into $L^{q}\left(\Omega_{2}, \mu_{2}\right)$.

Lemma B. Let $1<q, q^{\prime}<\infty$ such that $\frac{1}{q}+\frac{1}{q^{\prime}}=1$ and $\omega \in \mathcal{W}_{\varepsilon, \delta}^{q}$; $\omega(r)=\chi^{\frac{1}{q^{\prime}}}(r) \nu^{-\frac{1}{q}}\left(r^{-1}\right)$. For $0 \leq \varepsilon<\delta<\infty$ and $a, b \in \mathbb{R}_{+}^{*}$ let us set

$$
R^{\varepsilon, \delta}(a, b)=\frac{\omega(a)}{\omega(b)} \min \left(\left(\frac{a}{b}\right)^{\varepsilon},\left(\frac{b}{a}\right)^{\delta}\right) .
$$

Then there exist $C>0$ and a measurable function $g: \mathbb{R}_{+} \rightarrow \mathbb{R}_{+}$such that

$$
\int_{0}^{\infty} R^{\varepsilon, \delta}(a, b) g^{q^{\prime}}(a) \frac{d a}{a} \leq C g^{q^{\prime}}(b) \quad \text { a.e. } b>0
$$

and

$$
\int_{0}^{\infty} R^{\varepsilon, \delta}(a, b) g^{q}(b) \frac{d b}{b} \leq C g^{q}(a) \quad \text { a.e. } a>0
$$

Proof. It suffices to take $g(r)=\chi^{\frac{1}{q q^{\prime}}}(r) \nu^{-\frac{1}{q q^{\prime}}}\left(r^{-1}\right)$.

The main idea in the proof of the above Theorem is based on the following lemmas.

Lemma 4.1. Let $\psi, \phi \in S_{*, 0}(\mathbb{X})$. Then

$$
\left\|\Delta_{x} \phi_{\rho}\right\|_{1} \leq C \min \left(1,\left(\frac{x}{\rho}\right)^{2}\right)
$$

Proof. Using the fact that $\left\|\Delta_{x} \phi\right\|_{1} \leq C x^{2}$ (see [11]) and the contraction property of the generalized translation operators we obtain

$$
\left\|\Delta_{x} \phi\right\|_{1} \leq C \min \left(1, x^{2}\right)
$$


On the other hand, from the expression of the kernel $W_{\alpha}$, one can see easily that

$$
\left\|\Delta_{x} \phi_{\rho}\right\|_{1}=\left\|\Delta_{\frac{x}{\rho}} \phi\right\|_{1}
$$

So it holds

$$
\left\|\Delta_{x} \phi_{\rho}\right\|_{1} \leq C \min \left(1,\left(\frac{x}{\rho}\right)^{2}\right) .
$$

The lemma is proved.

Lemma 4.2. Let $\psi, \phi \in S_{*, 0}(\mathbb{X})$. Then

$$
\left\|\phi_{\rho \#} \psi_{r}\right\|_{1} \leq C \min \left(\left(\frac{r}{\rho}\right)^{2},\left(\frac{\rho}{r}\right)^{2}\right), \quad \text { for all } r, \rho>0 .
$$

Proof. Let us take $r \leq \rho$. Since $\int_{\mathbb{X}} \phi_{\rho}(y) d \mu_{\alpha}(y)=0$ we have

$$
\phi_{\rho \#} \psi_{r}(y)=\int_{\mathbb{X}} \psi_{r}(x) \Delta_{x} \phi_{\rho}(y) d \mu_{\alpha}(x) .
$$

From Minkowski's inequality one gets

$$
\begin{aligned}
\left\|\psi_{r \#} \phi_{\rho}\right\|_{1} & \leq \int_{\mathbb{X}}\left|\psi_{r}(x)\right|\left\|\Delta_{x} \phi_{\rho}\right\|_{1} d \mu_{\alpha}(x) \\
& =C \int_{\mathbb{X}}\left(\frac{x}{r}\right)^{2 \alpha+2}\left|\psi\left(\frac{x}{r}\right)\right|\left\|\Delta_{x} \phi_{\rho}\right\|_{1} \frac{d x}{x} .
\end{aligned}
$$

Taking into account that $\psi$ belongs to $S_{*}(\mathbb{X})$, then there exists $C>0$ such that

$$
\left(\frac{x}{r}\right)^{2 \alpha+2}\left|\psi\left(\frac{x}{r}\right)\right| \leq C\left(\frac{x}{r}\right)^{-3} \text { and }\left|\psi\left(\frac{x}{r}\right)\right| \leq C .
$$

So we get

$$
\left(\frac{x}{r}\right)^{2 \alpha+2}\left|\psi\left(\frac{x}{r}\right)\right| \leq C \min \left(\left(\frac{x}{r}\right)^{2 \alpha+2},\left(\frac{r}{x}\right)^{3}\right) .
$$

Hence from (4.6), (4.7) and (4.4) we obtain

$$
\left\|\psi_{r \#} \phi_{\rho}\right\|_{1} \leq C \int_{0}^{\infty}\left(\frac{x}{\rho}\right)^{2} \min \left(\left(\frac{x}{r}\right)^{2 \alpha+2},\left(\frac{r}{x}\right)^{3}\right) \frac{d x}{x}=C\left(\frac{r}{\rho}\right)^{2} .
$$

The lemma is proved.

One of the main tool used in this paper is the Calderón reproducing formula that we recall in the following lemma (see [17]). 
Lemma 4.3. Let $\psi \in S_{*, 0}^{1}$, and $1<\varepsilon<\infty$. For $f \in L^{p}\left(d \mu_{\alpha}\right)(1 \leq p<$ $\infty)$ define

$$
f_{\varepsilon}=\int_{1 / \varepsilon}^{\varepsilon} f_{\#} \psi_{r \#} \psi_{r} \frac{d r}{r} .
$$

Then $f_{\varepsilon}$ converges to $f$ in $L^{p}\left(d \mu_{\alpha}\right)$ as $\varepsilon \rightarrow \infty$.

Lemma 4.4. (i) Let $1 \leq p \leq \infty$ and $\psi \in S_{*, 0}$. Then we have for all $\beta>0$ and $f$ such that $\mathbf{B}_{p, q}^{\omega}(f)<\infty$

$$
\left\|f_{\#} \psi_{r}\right\|_{p} \leq C \int_{\mathbb{X}} \min \left(\left(\frac{x}{r}\right)^{2 \alpha+2},\left(\frac{r}{x}\right)^{\beta}\right)\left\|\Delta_{x} f\right\|_{p} \frac{d x}{x}, \quad \text { a.e. } \quad r>0 .
$$

(ii) For all $f \in \Lambda_{p, q}^{\omega}(\mathbb{X})$ it holds

$$
\left\|\Delta_{x} f\right\|_{p} \leq C \int_{0}^{\infty} \min \left(1,\left(\frac{x}{r}\right)^{2}\right)\left\|f_{\#} \psi_{r}\right\|_{p} \frac{d r}{r}, \quad \text { a.e. } x \in \mathbb{X} .
$$

Proof. The result follows from Lemmas 4.1, 4.2 and 4.3.

Proof of Theorem 4.1. (i) Let us start with the case $q=\infty$ which follows immediately from (4.10) and (4.11). Assume $f$ such that $\mathbf{B}_{p, \infty}^{\omega}(f)<\infty$. Using (4.10) with $\beta \geq 2$, we get

$$
\begin{aligned}
\left\|f_{\#} \psi_{r}\right\|_{p} & \leq C \int_{\mathbb{X}} \min \left(\left(\frac{x}{r}\right)^{2 \alpha+2},\left(\frac{r}{x}\right)^{\beta}\right)\left\|\Delta_{x} f\right\|_{p} \frac{d x}{x} \\
& \leq C \mathbf{B}_{p, \infty}^{\omega}(f) \int_{\mathbb{X}} \omega(x) \min \left(\left(\frac{x}{r}\right)^{2 \alpha+2},\left(\frac{r}{x}\right)^{\beta}\right) \frac{d x}{x} \\
& \leq C \mathbf{B}_{p, \infty}^{\omega}(f) \omega(r) .
\end{aligned}
$$

That is $\mathbf{A}_{p, \infty}^{\omega}(f) \leq C \mathbf{B}_{p, \infty}^{\omega}(f)$.

Take now $f \in \Lambda_{p, \infty}^{\omega}(\mathbb{X})$. Then, from (4.11) it holds

$$
\begin{aligned}
\left\|\Delta_{x} f\right\|_{p} & \leq C \int_{0}^{\infty} \min \left(1,\left(\frac{x}{r}\right)^{2}\right)\left\|f_{\#} \psi_{r}\right\|_{p} \frac{d r}{r} \\
& \leq C \mathbf{A}_{p, \infty}^{\omega}(f) \int_{0}^{\infty} \omega(r) \min \left(1,\left(\frac{x}{r}\right)^{2}\right) \frac{d r}{r} \\
& \leq C \mathbf{A}_{p, \infty}^{\omega}(f) \omega(x) .
\end{aligned}
$$

(ii) Let us prove the case $q=1$. Assume $f$ such that $\mathbf{B}_{p, 1}^{\omega}(f)<\infty$. We shall prove that $\mathbf{A}_{p, 1}^{\omega}(f) \leq C \mathbf{B}_{p, 1}^{\omega}(f)$. From (4.10), with $\beta \geq 2$ we obtain

$$
\mathbf{A}_{p, 1}^{\omega}(f)=\int_{0}^{\infty} \frac{\left\|f_{\#} \psi_{r}\right\|_{p}}{\omega(r)} \frac{d r}{r}
$$




$$
\begin{aligned}
& \leq C \int_{0}^{\infty} \frac{1}{\omega(r)} \int_{\mathbb{X}} \min \left(\left(\frac{x}{r}\right)^{2 \alpha+2},\left(\frac{r}{x}\right)^{\beta}\right)\left\|\Delta_{x} f\right\|_{p} \frac{d x}{x} \frac{d r}{r} \\
& =C \int_{\mathbb{X}}\left\|\Delta_{x} f\right\|_{p} \int_{0}^{\infty} \frac{1}{\omega(r)} \min \left(\left(\frac{x}{r}\right)^{2 \alpha+2},\left(\frac{r}{x}\right)^{\beta}\right) \frac{d r}{r} \frac{d x}{x} \\
& \leq C \int_{\mathbb{X}} \frac{\left\|\Delta_{x} f\right\|_{p}}{\omega(x)} \frac{d x}{x} \\
& =C \mathbf{B}_{p, 1}^{\omega}(f) .
\end{aligned}
$$

Conversely, let us take $f$ in $\Lambda_{p, 1}^{\omega}(\mathbb{X})$. Then using (4.11), we get

$$
\begin{aligned}
\mathbf{B}_{p, 1}^{\omega}(f) & =\int_{\mathbb{X}} \frac{\left\|\Delta_{x} f\right\|_{p}}{\omega(x)} \frac{d x}{x} \\
& \leq C \int_{\mathbb{X}} \int_{0}^{\infty} \frac{1}{\omega(x)} \min \left(1,\left(\frac{x}{r}\right)^{2}\right)\left\|f_{\#} \psi_{r}\right\|_{p} \frac{d r}{r} \frac{d x}{x} \\
& =C \int_{0}^{\infty}\left\|f_{\#} \psi_{r}\right\|_{p} \int_{\mathbb{X}} \frac{1}{\omega(x)} \min \left(1,\left(\frac{x}{r}\right)^{2}\right) \frac{d x}{x} \frac{d r}{r} \\
& \leq C \int_{0}^{\infty} \frac{\left\|f_{\#} \psi_{r}\right\|_{p}}{\omega(r)} \frac{d r}{r} \\
& =C \mathbf{A}_{p, 1}^{\omega}(f) .
\end{aligned}
$$

(iii) Let us now prove the case $1<q<\infty$. Using the inequality (4.10) we obtain, for $\beta \geq 2$

$$
\begin{aligned}
\frac{\left\|f_{\#} \psi_{r}\right\|_{p}}{\omega(r)} & \leq C \int_{\mathbb{X}} \frac{\omega(x)}{\omega(r)} \min \left(\left(\frac{x}{r}\right)^{2 \alpha+2},\left(\frac{r}{x}\right)^{\beta}\right) \frac{\left\|\Delta_{x} f\right\|_{p}}{\omega(x)} \frac{d x}{x} \\
& =C \int_{\mathbb{X}} R^{2 \alpha+2, \beta}(x, r) \frac{\left\|\Delta_{x} f\right\|_{p}}{\omega(x)} \frac{d x}{x} \\
& =C T_{R^{2 \alpha+2, \beta}}\left(\frac{\left\|\Delta_{x} f\right\|_{p}}{\omega(x)}\right)(r)
\end{aligned}
$$

where $R^{2 \alpha+2, \beta}(x, r)=\frac{\omega(x)}{\omega(r)} \min \left(\left(\frac{x}{r}\right)^{2 \alpha+2},\left(\frac{r}{x}\right)^{\beta}\right)$. By Lemma A and Lemma $\mathrm{B}$, we obtain the boundedness of the operator $T_{R^{2 \alpha+2, \beta}}$ from $L^{q}\left(\frac{d x}{x}\right):=$ $L^{q}\left(\mathbb{X}, \frac{d x}{x}\right)$ into $L^{q}\left(\frac{d r}{r}\right):=L^{q}\left(\mathbb{R}_{+}^{*}, \frac{d r}{r}\right)$. Moreover the hypothesis $\mathbf{B}_{p, q}^{\omega}(f)<$ $\infty$ means that the function $x \longmapsto \frac{\left\|\Delta_{x} f\right\|_{p}}{\omega(x)}$ belongs to $L^{q}\left(\frac{d x}{x}\right)$. Hence

$$
\begin{aligned}
\mathbf{A}_{p, q}^{\omega}(f) & =\left\|\frac{\left\|f_{\#} \psi_{r}\right\|_{p}}{\omega(r)}\right\|_{L^{q}\left(\frac{d r}{r}\right)} \leq C\left\|T_{F}\left(\frac{\left\|\Delta_{x} f\right\|_{p}}{\omega(x)}\right)\right\|_{L^{q}\left(\frac{d r}{r}\right)} \\
& \leq C\left\|\frac{\left\|\Delta_{x} f\right\|_{p}}{\omega(x)}\right\|_{L^{q}\left(\frac{d x}{x}\right)}=C \mathbf{B}_{p, q}^{\omega}(f) .
\end{aligned}
$$


Conversely, let us take $f \in \Lambda_{p, q}^{\omega}(\mathbb{X})$. From (4.11) we get

$$
\begin{aligned}
\frac{\left\|\Delta_{x} f\right\|_{p}}{\omega(x)} & \leq C \int_{0}^{\infty} \frac{\omega(r)}{\omega(x)} \min \left(1,\left(\frac{x}{r}\right)^{2}\right) \frac{\left\|f_{\#} \psi_{r}\right\|_{p}}{\omega(r)} \frac{d r}{r} \\
& =C \int_{0}^{\infty} R^{0,2}(r, x) \frac{\left\|f_{\#} \psi_{r}\right\|_{p}}{\omega(r)} \frac{d r}{r} \\
& =C T_{R^{0,2}}\left(\frac{\left\|f_{\#} \psi_{r}\right\|_{p}}{\omega(r)}\right)(x)
\end{aligned}
$$

where $R^{0,2}(r, x)=\frac{\omega(r)}{\omega(x)} \min \left(1,\left(\frac{x}{r}\right)^{2}\right)$. We proceed as above with adequate changes to obtain

$$
\begin{aligned}
\mathbf{B}_{p, q}^{\omega}(f) & \leq C\left\|T_{R^{0,2}}\left(\frac{\left\|f_{\#} \psi_{r}\right\|_{p}}{\omega(r)}\right)\right\|_{L^{q}\left(\frac{d x}{x}\right)} \\
& \leq C\left\|\frac{\left\|f_{\#} \psi_{r}\right\|_{p}}{\omega(r)}\right\|_{L^{q}\left(\frac{d r}{r}\right)} \\
& =C \mathbf{A}_{p, q}^{\omega}(f)
\end{aligned}
$$

which gives the desired result.

Theorem 4.2 (Second characterization theorem). Let $1 \leq p, q \leq \infty$ and $\omega \in \mathcal{W}_{0,2}^{q}$. For $f$ in $\Lambda_{p, q}^{\omega}(\mathbb{X})$ put



where $m_{p}(f, x)=\sup _{0 \leq y \leq x}\left\|\Delta_{y} f\right\|_{p}$ is the generalized modulus of continuity associated with the generalized translation operators $T_{x}^{(\alpha)}$. Then $\mathbf{C}_{p, q}^{\omega}$ is a norm on $\Lambda_{p, q}^{\omega}(\mathbb{X})$ equivalent to $\mathbf{A}_{p, q}^{\omega}$.

Proof. To compare $\mathbf{A}_{p, q}^{\omega}(f)$ and $\mathbf{C}_{p, q}^{\omega}(f)$ we proceed as in the proof of the Theorem 4.1 using the following lemma instead of Lemma 4.4.

Lemma 4.5. (i) Let $1 \leq p \leq \infty$ and $\psi \in S_{*, 0}$. Then we have for all $\beta>0$ and $f$ such that $\mathbf{B}_{p, q}^{\omega}(f)<\infty$

$$
\left\|f_{\#} \psi_{r}\right\|_{p} \leq C \int_{\mathbb{X}} \min \left(\left(\frac{x}{r}\right)^{2 \alpha+2},\left(\frac{r}{x}\right)^{\beta}\right) m_{p}(f, x) \frac{d x}{x}, \quad \text { a.e. } \quad r>0 .
$$


(ii) For all $f \in \Lambda_{p, q}^{\omega}(\mathbb{X})$ it holds

$$
m_{p}(f, x) \leq C \int_{0}^{\infty} \min \left(1,\left(\frac{x}{r}\right)^{2}\right)\left\|f_{\#} \psi_{r}\right\|_{p} \frac{d r}{r}, \quad \text { a.e. } x \in \mathbb{X} .
$$

\section{References}

[1] G. Altenburg, Eine realisierung der theorie der abstrakten Besovräume $B_{q}^{s}(A) \quad(s>0,1 \leq q \leq \infty)$ und der Lebesgue-Räume $H_{p, \mu}^{s}$ auf der Grundlage besselscher differentialoperatoren, Z. Analysis u. Anwendungen, 3 (1984), 43-63.

[2] J. L. Ansorena and O. Blasco, Characterization of weighted Besov spaces, Math. Nachr., 171 (1995), 5-17.

[3] M. Assal and H. Ben Abdallah, Generalized Besov type spaces on the Laguerre hypergroup, Annales Mathématiques Blaise Pascal, 12 (2005), 117-145.

[4] O. V. Besov, On a family of function spaces in connection with embeddings and extensions, Trudy Mat. Inst. Steklov, 60 (1961), 4281.

[5] J. J. Betancor and L. Rodríguez-Mesa, On the Besov-Hankel spaces, J. Math. Soc. Japan, 50 (1998), 781-788.

[6] H. Bahouri, P. Gérard and C. J. Xu, Espaces de Besov et estimations de Strichartz généralisées sur le groupe de Heisenberg, Journal d'Analyse Mathématique, 82 (2000).

[7] W. R. Bloom and H. Heyer, Harmonic analysis of probability measures on hypergroups, de Gruyter, Studies in Mathematics, Vol. 20, de Gruyter, Berlin, New York, 1994.

[8] G. Bourdaud, $R$ ealisation des espaces de Besov homogènes, Arkiv för Mat., 26 (1988), 41-54.

[9] J.-Y. Chemin, About Navier-Stokes system, Publication du Laboratoire d'Analyse Numerique, Université Pierre et Marie Curie, 1996.

[10] R. I. Jewett, Spaces with an abstract convolution of measures, Adv. in Math., 18 (1975), 1-101.

[11] J. Löfström and J. Peetre, Approximation theorems connected with generalized translations, Math. Ann., 181 (1969), 255-268.

[12] J. Peetre, New thoughts on Besov spaces, Duke Univ. Math. Serie Durham, Univ., 1976. 
[13] H. Schmeisser and J.-H. Triebel, Topics in Fourier Analysis and Function Spaces, Wiley, Chichester, 1987.

[14] H. Triebel, Theory of Function Spaces II, Birkhaüser, 1992.

[15] H. Triebel, Fourier analysis and function spaces, Teubner Math., 7, Teubner, Leipzig, 1977.

[16] K. Trimèche, Generalized Harmonic Analysis and Wavelet Packets, Gordon and Breach Science Publishers, Australia, 2001.

[17] K. Trimèche and M. A. Mourou, Calderón's reproducing formula associated with the Bessel operator, JMAA, 219 (1998), 97-109.

Département de Mathématiques IPEIN

Campus Universitaire Mrezka 8000

Nabeul

Tunisia

(E-mail : Miloud.Assal@fst.rnu.tn)

Département de Mathématiques

Faculté des Sciences de Bizerte

Zarzouna 7021

Bizerte

Tunisia

(E-mail : Hacen.Benabdallah@fst.rnu.tn) 


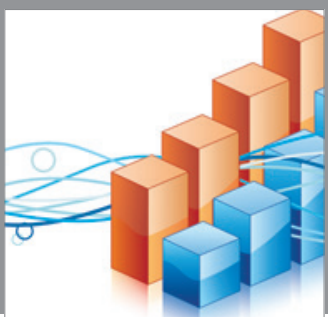

Advances in

Operations Research

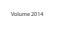

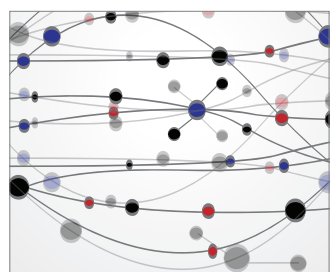

\section{The Scientific} World Journal
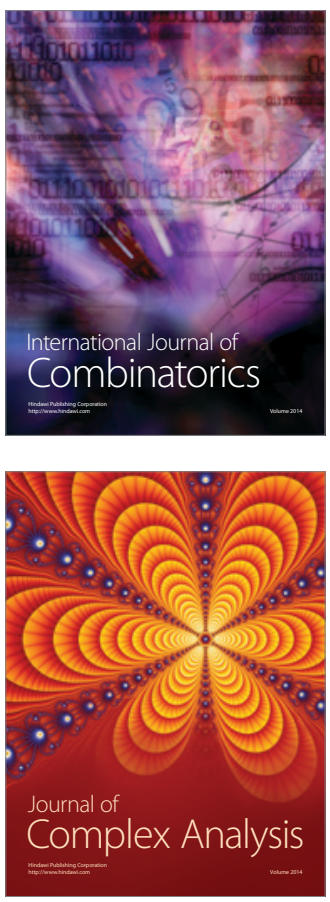

International Journal of

Mathematics and

Mathematical

Sciences
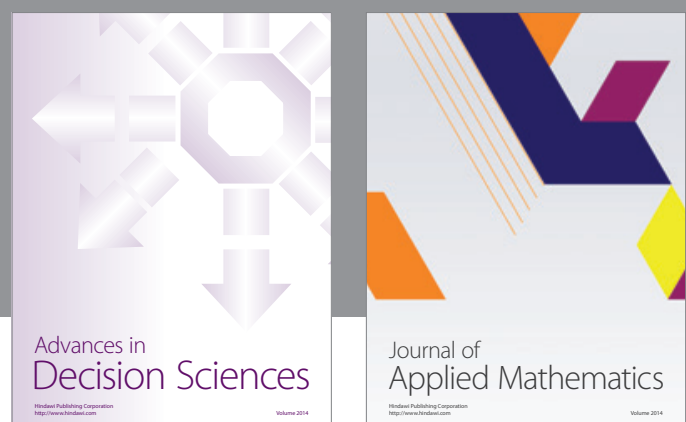

Journal of

Applied Mathematics
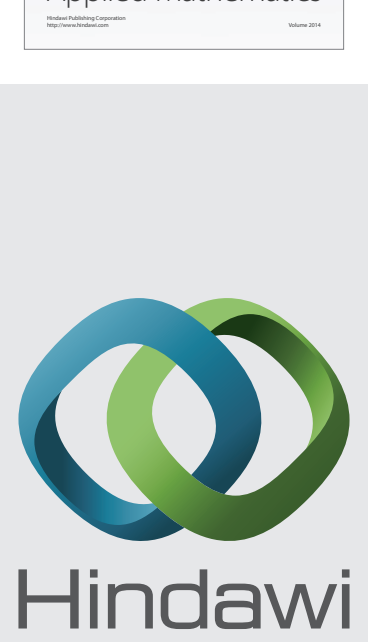

Submit your manuscripts at http://www.hindawi.com
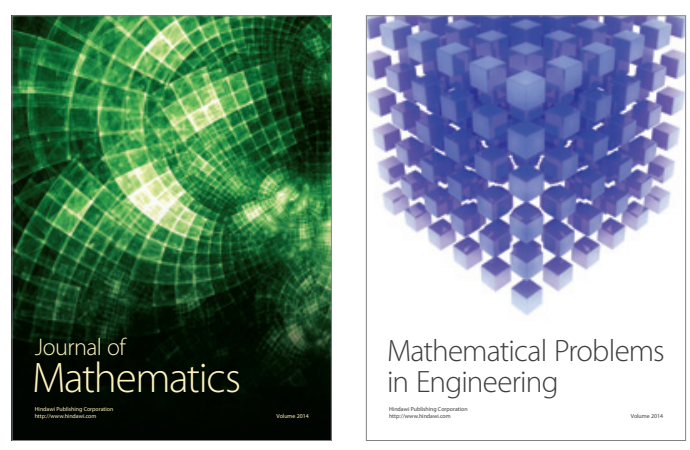

Mathematical Problems in Engineering
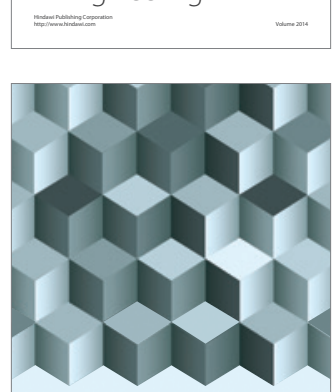

Journal of

Function Spaces
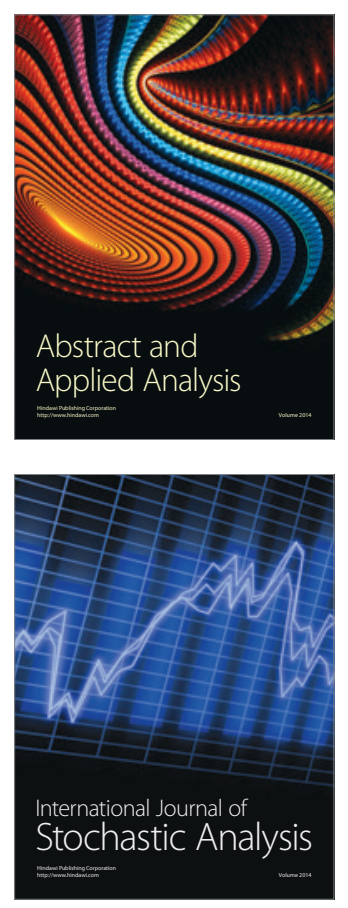

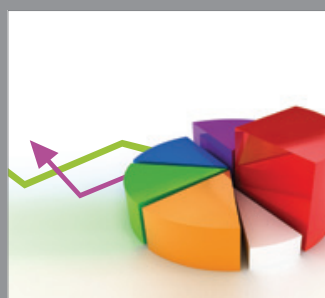

ournal of

Probability and Statistics

Promensencen
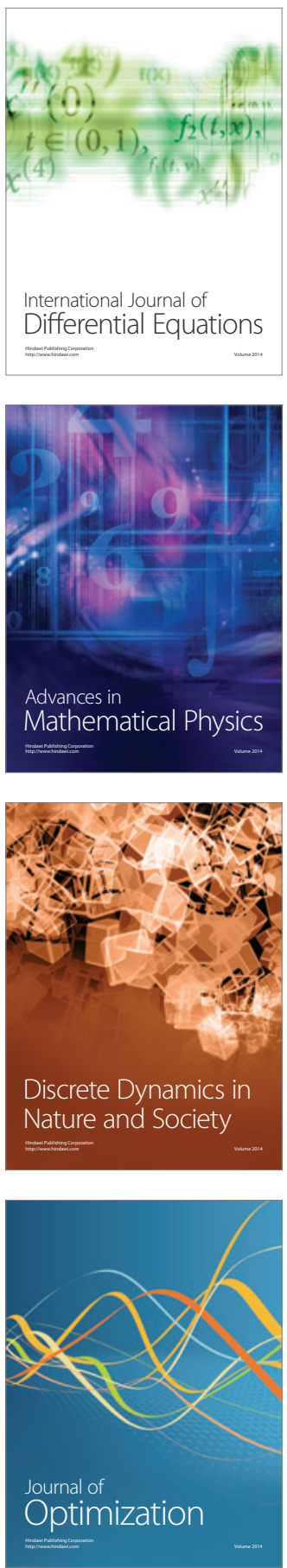